\title{
Androgen-Induced Changes in Electrocommunicatory Behavior Are Correlated with Changes in Substance P-like Immunoreactivity in the Brain of the Electric Fish Apteronotus leptorhynchus
}

\author{
Joseph G. Dulka, Leonard Maler, and William Ellis \\ Department of Anatomy and Neurobiology, University of Ottawa, Faculty of Medicine, Ottawa, Ontario, Canada \\ $\mathrm{K} 1 \mathrm{H} 8 \mathrm{M} 5$
}

The hormonal regulation of sex differences in electrocommunicatory behavior and brain substance P-like immunoreactivity (SPI-ir) were examined in the weakly electric fish, Apteronotus leptorhynchus. This animal modulates its electric organ discharge (EOD) to produce discrete electric social signals (chirps), which function in aggressive and reproductive displays. Males readily chirp in response to electrosensory stimuli that mimic the presence of a conspecific; females also chirp in response to such stimuli, but do so at much lower rates than males. We have recently demonstrated that androgen treatment enhances chirping behavior in females and may also lead to a change in chirp quality or structure. In this study, we quantified androgen-induced changes in chirp structure and simultaneously examined whether androgens alter the sexually dimorphic pattern of SPI-ir in a brain region (prepacemaker nucleus, PPn) known to control chirping. Our results demonstrate that, in females, androgens cause both the induction of chirping and an alteration of chirp structure; chirps recorded from androgen-implanted females had longer durations and more dramatic frequency and amplitude modulations compared to controls, and appear similar to those reported to be produced during spawning. Moreover, androgen-induced changes in chirping are correlated with increased expression of SPI-ir within specific brain nuclei of females. These changes may underly behavioral changes in chirping, since treated females showed a male-like pattern of SPI-ir in the PPn. However, alterations in SPI-ir were not restricted to the PPn, but also occurred in diencephalic regions related to pituitary function and reproductive behavior. The results suggest that androgens modulate chirping activity and cause both specific and wide-spread changes in SPI-ir that may relate to a functional system that interrelates pituitary function, reproductive behavior, and chirping.

[Key words: neuronal plasticity, steroid hormones, reproductive behavior, electrocommunication, chirping behavior, electric fish]

\footnotetext{
Received May 23, 1994; revised July 25, 1994; accepted Aug. 30, 1994

This work was supported by a postdoctoral fellowship from the Medical Research Council of Canada (MRC) to J.G.D., and an MRC grant to L.M. We thank Dr. Glen Van Der Kraak, University of Guelph, for determining plasma levels of T by RIA. J.G.D. also thanks Dr. Martin Alda and Ms. Eliane Gaude for their support during the preparation of the manuscript.

Correspondence should be addressed to Dr. Joseph G. Dulka, Department of Biology, Creighton University, 2500 California Plaza, Omaha, NE 68178-0103. Copyright (C) 1995 Society for Neuroscience $0270-6474 / 95 / 151879-12 \$ 05.00 / 0$
}

Hormonally sensitive, sexually dimorphic neural systems are found in variety of vertebrates, including humans (Breedlove, 1992). In many cases, sex differences in reproductive behavior can be attributed to organizational or activational effects of gonadal steroids and their interplay with sexually dimorphic brain regions (Macl usky and Noftolin, 1981; McEwen, 1981). Many of the actions of gonadal steroids are mediated by effects on neurotransmitter or neuropeptide systems that regulate reproductive behaviors (Hutchison, 1991). Vertebrate neural systems that display prominent sexual dimorphisms include the songbird vocal control system, the frog vocalization system, the rodent spinal-bulbocavernosus copulatory system, and the more generalized sexually dimorphic nucleus of the preoptic area (see review by Breedlove, 1992).

This report concerns another vertebrate system that shows prominent sex differences; namely, the production of electric social signals by the weakly electric fish Apteronotus leptorhynchus. This teleost system is particularly attractive as a neuroethological model for the study of brain-hormone interactions, since it is one of the few vertebrate examples in which a complex behavior can be explained in terms of its underlying neural circuitry (Kawasaki et al., 1988).

A. leptorhynchus emits a continuous weak electric organ discharge (EOD), which is used for both electrolocation and intraspecific communication (Bullock and Heiligenberg, 1986). These animals can modulate their EOD to produce discrete social signals, or chirps, which are characterized by a brief increase in EOD frequency and a simultaneous decrease in EOD amplitude. Chirps are produced only during the performance of agonistic or reproductive behaviors. However, the types of chirps produced under agonistic and reproductive conditions differ with respect to their structure or quality (Hopkins, 1974; Hagedorn and Heiligenberg, 1985). Both males and females chirp during courtship and spawning (Hagedorn and Heiligenberg, 1985; Hagedorn, 1986), and the duration of courtship chirps may be up to 10 times longer than chirps produced during aggression; the longer courtship chirps are characterized by more dramatic frequency and amplitude modulations (Hagedorn and Heiligenberg, 1985).

A. leptorhynchus will also produce chirps in response to a sinusoidal signal that mimics the presence of a conspecific; such signals are normally delivered to the water at frequencies $1-10$ $\mathrm{Hz}$ above or below an animal's own discharge frequency (Dye, 1987). However, when tested under these conditions, males and females show clear differences in their propensity to chirp; males readily chirp in response to appropriate electrosensory 
stimuli, whereas females generally do not (Dye, 1987; Dulka and Maler, 1994; Zupanc and Maler, 1994). We have recently demonstrated that testosterone implants cause an enhancement in female chirping behavior and may also lead to changes in chirp structure related to reproduction (Dulka and Maler, 1994).

A previous study demonstrated that substance P-like immunoreactivity (SPl-ir) is sexually dimorphic in the brain of $A$. leptorhynchus. This peptide is present in a number of diencephalic nuclear groups of males but not females (Weld and Maler, 1992). One of these brain regions, the prepacemaker nucleus (PPn), is the command center for chirping behavior (Kawasaki et al., 1988).

The objectives of the present study were threefold. First, to extend our previous findings, we quantify androgen-induced changes in chirp structure. Second, to address the possible mechanism of hormone action, we tested the effects of aromatizable (testosterone, T) and nonaromatizable (dihydrotestosterone, DHT) androgens on chirp structure. Third, because substance $P$ has been implicated in the control of chirping behavior (Weld and Maler, 1991), we examined whether androgen treatment causes changes in SPl-ir in the vicinity of the PPn.

\section{Materials and Methods}

Animals. Brown Ghost knife fish (Apteronotus leptorhynchus), ranging in size from 16.0 to $18.5 \mathrm{~cm}$, were purchased from Tropical Fish Suppliers (Fort Erie, Ontario), and maintained in large (200 l) community aquaria on a $12 \mathrm{~L}: 12 \mathrm{D}$ photoperiod. All fish were fed live black worms, ad libitum, at least twice a week.

Behavioral testing. Fish to be used for behavioral testing were transferred to smaller test aquaria (20 1) which contained gravel substrate, a submersible heater $\left(27-28^{\circ} \mathrm{C}\right)$, an air-powered box filter, and a modified plastic tube that would accommodate the insertion of a restraining apparatus (Dulka and Maler, 1994). This species is nocturnal, and fish generally reside in plastic tubes during the day. This behavior greatly facilitated the testing of individual fish in their home aquaria without handling the animal prior to testing.

In order to distinguish males from females, it was necessary to first screen the animals for their propensity to chirp in response to a synthetic sine wave at a frequency $3.0 \mathrm{~Hz}$ below the fish's own discharge frequency. Both sexes will readily perform the jamming avoidance response (JAR) in response to a $3 \mathrm{~Hz}$ below stimulus (Heiligenberg, 1991); males consistently chirp in response to this stimulus, whereas females generally do not (Dulka and Maler, 1994; Zupanc and Maler, 1994). The screening procedure (and all subsequent experimental testing) was performed on individual fish in home aquaria by sliding the restraining apparatus into position, thereby temporarily trapping the fish within its tube. Once in position, the apparatus allowed for simultaneous monitoring of the fish's own EOD and stimulus delivery. The fish's EOD signal was recorded along the long axis of the body by two silver electrodes embedded in the ends of the restraining apparatus. To monitor changes in EOD frequency, the animal's EOD signal was amplified (Grass P15D AC preamplifier), broadcast on an audio monitor, displayed on an oscilloscope (Tektronics T912), and fed into a frequency to voltage converter (PTR 1000; PolyTek Research) which drove a Grass $79 \mathrm{D}$ physiograph. The stimulus signal, produced by a sign-wave generator (Wavetek model 186; Wavetek, San Diego, Inc.), was passed through a photo isolation unit, and delivered across the fish's body through two carbon electrodes held in position by the testing apparatus. The stimulus signal was adjusted to yield a field intensity of $1.0 \mathrm{mV} / \mathrm{cm}$, as measured from the midpoint hetween the two stimulating electrodes. The screening procedure began by recording the animal's basal EOD for at least $30 \mathrm{sec}$ prior to delivering the stimulus signal $(3 \mathrm{~Hz}$ below the animal's own EOD) for an additional $30 \mathrm{sec}$. The occurrence of both the JAR and evoked chirping behavior were monitored during two consecutive test periods, separated by at least $1 \mathrm{~min}$ of basal EOD recording. Only animals that responded by performing clear JARs without chirping were considered to be females. Moreover, only animals that met this criteria over 3 consecutive screening days were used in the androgen implant experiments.

Hormone treatments. This experiment tested the effects of testoster- one (T) and dihydrotestosterone (DHT) on female chirping behavior in A. leptorhynchus. The procedures used for behavioral testing and hormone manipulation were identical to those described by Dulka and Maler (1994). Briefly, all animals were tested using stimuli $3 \mathrm{~Hz}$ below and $10 \mathrm{~Hz}$ above the animal's own EOD frequency on day 0 to establish pretreatment values for the incidence of evoked chirping. For each female, both forms of stimuli were tested twice on a given day, and the resulting behavioral responses were averaged to obtain daily values for chirping behavior. Besides being recorded on the physiograph, all behavioral responses were digitized (Neuro-Corder DR-390; Neurodata Instruments Corp., $44 \mathrm{kHz}$ sampling rate) and stored on video tape (Sony SLV-M88; Sony Corp.) for off-line computer analysis.

On day 1 , the animals were randomly assigned to control and experimental groups and implanted intraperitoneally with either solid SILASTIC pellets $(0.5 \mathrm{~mm} / \mathrm{gm}$ body weight) alone (controls) or solid SILASTIC pellets containing sufficient T or DHT to create a final dose of $100 \mu \mathrm{g} / \mathrm{g}$ body weight. Most of the animals were then tested for their propensity to chirp on postimplant days 15 and 30; a subset of each group was also tested on day 45 . As a positive control, six nonimplanted males were also tested in parallel with the females. At the end of the cxpcriment, all animals were ancsthetized by immersion in $0.05 \%$ 2-phenoxyethanol (Syndel Laboratories), bled with heparinized syringes through the caudal vasculature, and perfused (see below). Following the removal of the brain, each fish was dissected to verify sex and gonadal maturity.

Substance P-like (SPl-ir) immunohistochemistry. All animals were perfused through the conus arteriosus with fixative containing $4 \%$ paraformaldehyde and $0.17 \%$ picric acid in $0.1 \mathrm{M}$ phosphate-buffered saline (PBS, $0.9 \% \mathrm{NaCl}$ ). Brains were postfixed in the same perfusion fixative for $1 \mathrm{hr}$, left overnight at $4^{\circ} \mathrm{C}$ in $15 \%$ sucrose-PBS, and processed for SPl-ir either immediately or following up to 1 month storage in a polyvinyl pyrrolidone-sucrose-ethylene glycol based cryoprotectant, which preserves immunogenicity in this species (Lannoo et al., 1991). All brains were rapidly frozen on dry ice, sectioned $\left(-20^{\circ} \mathrm{C}\right)$ on a cryostat $(15 \mu \mathrm{m})$, and mounted on chrome alum-subbed slides. The brains of fish sacrificed after day 45 were serially sectioned throughout the entire forebrain; the brains of fish sacrificed after day 30 were sectioned through the diencephalic PPn region only. In each instance, at least one brain from each of the experimental groups (i.e., blank-implanted control, T-implanted, and DHT-implanted females) were processed together for SPl-ir. In addition, the brains of the six normal males were processed in parallel with those of the females in order to provide a positive control for the sexually dimorphic pattern of SPl-ir observed in this species (Weld and Maler, 1992).

The immunohistochemical procedure used in this study was similar to that described by Weld and Maler (1992). Briefly, sections were rinsed twice in PBS for $15 \mathrm{~min}$ and incubated for $24 \mathrm{hr}$ at $4^{\circ} \mathrm{C}$ with rabbit SP antiserum (1:1000, Amersham RPN 1572) in PBS containing $0.3 \%$ Triton X-100 (TX) and $1 \%$ bovine serum albumin (PBS-TXBSA). The specificity of this antisera has been previously established for A. leptorhynchus (Weld and Maler, 1992). Following two rinses in PBS for a total of $15 \mathrm{~min}$, the sections were incubated for $2 \mathrm{hr}$ at room temperature with biotinylated donkey anti-rabbit antiserum (Amersham RPN 1004) at a dilution of 1:200 in PBS-TX-BSA. The sections were again rinsed twice in PBS for $15 \mathrm{~min}$ and incubated for $1 \mathrm{hr}$ at room temperature with either horseradish peroxidase-labeled streptavidin (Amersham RPN 1231, 1:200) or florescent CY3-labeled streptavidin (Sigma, 1:100) in PBS alone. The horseradish peroxidase-labeled sections were rinsed in PBS and transferred to $0.1 \mathrm{M}$ sodium acetate buffer (SAB, pH 6.0) for $5 \mathrm{~min}$ and then preincubated for $5 \mathrm{~min}$ in $100 \mathrm{ml}$ of SAB containing $0.02 \%$ diaminobenzidine (Sigma), $0.6 \%$ nickel ammonium sulfate $(\mathrm{BDH})$, and $0.04 \%$ ammonium chloride (Sigma). The slides were briefly removed and $15 \mu \mathrm{l}$ of $30 \%$ hydrogen peroxide was added to the solution before reintroducing the sections for $15 \mathrm{~min}$. The peroxidase reaction was stopped by multiple washes in SAB and the sections were then dehydrated in an alcohol series, cleared in xylene, and coverslipped (Permount). The CY3-labeled sections were rinsed in multiple washes in PBS and coverslipped using a glycerol-based mounting medium. All immunoreacted sections were viewed and photographed on an Olympus BH-2 microscope.

Quantification of SPl-ir staining in the PPn. SPI-ir in the region of the ventral dendritic territory of the PPn (PPn-VT, Kawasaki et al., 1988) was examined in brains from 8 control females, $8 \mathrm{~T}$-implanted females, 11 DHT-implanted females, and 6 normal males. In all sections, the PPn-VT region was viewed at a magnification of $400 \times$ on a 
Zeiss microscope mounted with a Sony XC-77 video camera interfaced to Macintosh IIci microcomputer. Image analysis software (Image, version $1.47, \mathrm{NIH}$ ) was used to quantify the number of SPl-ir particles within the PPn-VT. In all cases, the images were displayed on the monitor, computer enhanced, and digitized before determining the number of SPI-ir particles present. The threshold for data acquisition and the limits (minimum and maximum) of particle size detection were held constant for all measurements. Unilateral measurements of SPl-ir in the PPn-VT were taken from three consecutive sections from each brain. These values were averaged to obtain individual values for each fish, which were then averaged within treatment groups.

Computer analysis of behaviorally evoked chirps. Chirp analysis was performed according to methods described by Zupanc and Maler (1994). Briefly, the EOD signals stored on video tape were first converted to analog signals and then redigitized at a sampling rate of 20 $\mathrm{kHz}$ using a MacAdios II computer board (GW Instruments) on a Macintosh Ilfx microcomputer. In order to capture only chirps (i.e., frequency and amplitude modulations of the EOD), the recorded EOD was simultaneously fed through a frequency-to-voltage converter that increased its output during a chirp and triggered a TTL pulse that initiated 200 misec of data capture (4000 data points), including $25 \mathrm{msec}$ of pretrigger data acquisition. The software (WAVETRAK, version 1.1; Ortex Systems) allowed most, if not all (see below), of the behaviorally evoked chirps to be captured within the standard $30 \mathrm{sec}$ stimulation period. This information was then stored on a hard disk for later analysis.

Wave analysis software (IGOR, version 1.26, WaveMetrics) was used for offline analysis of 323 chirps recorded from control $(n=47)$ and androgen treated $(n=204)$ females as well as six representative males $(n=72)$. The procedures used were similar to those described by $\mathrm{Zu}$ panc and Maler (1994). The times of positive-going zero crossings of the EOD were determined and used to compute the instantancous EOD frequency as a function of time. Five chirp parameters were then extracted from each chirp: (1) the peak EOD frequency increase during the chirp (FREQ), defined as the peak frequency during a chirp minus the average frequency during the first 10 EOD cycles of the $200 \mathrm{msec}$ recording; (2) the average EOD frequency increase during the chirp (AVFREQ), defined as the average frequency during a chirp minus the average frequency during the first 10 EOD cycles of the $200 \mathrm{msec}$ recording; (3) the relative EOD amplitude change during the chirp (AMP), defined as difference between the minimum amplitude during a chirp and the average amplitude of the first 10 cycles divided by the average amplitude (first 10 cycles); because relative chirp amplitude is expressed as ratio, it could range from 0 (no decrease in EOD amplitude during a chirp) to 1 (EOD amplitude goes to 0 during a chirp); (4) the absolute EOD amplitude change during the chirp (ABAMP), defined as the difference between the maximum and minimum amplitudes associated with a chirp; and (5) the duration of the chirp (DUR), defined as the time during which the chirp frequency remained $10 \%$ of its maximal above the baseline frequency (first 10 cycles).

Hormone assay. Blood samples were placed on chipped ice, centrifuged, and the resulting plasma stored at $-80^{\circ} \mathrm{C}$. Blood levels of $\mathrm{T}$ were determined by radioimmunoassay (RIA), using an antiserum specific for T (Van Der Kraak et al., 1984). However, because this antiserum also exhibits a $25 \%$ cross-reactivity with DHT (G. Van Der Kraak, personal communication), the RIA can be used to determine if circulating levels of immunoreactive "androgens" are elevated in DHTimplanted animals relative to blank-implanted controls.

Statistical analysis. All statistical analyses were performed using SYsTAT (version 5.1, SYSTAT, Inc.) on a Macintosh computer. All behavioral data were first analyzed by repeated measures ANOVA to determine if significant differences existed between treatment groups over time. Differences in the propensity to chirp between control and androgen-treated groups on a particular day were determined by independent $t$ tests. Differences between $\mathbf{T}$ concentrations in control and androgentreated animals were determined by independent $t$ tests. Differences in SPl-ir staining in the PPn-VT region of treated and nontreated females and males were determined by $\Lambda$ NOV $\Lambda$ and independent $t$ tests. Tests for normality of distribution of control and androgen-treated chirp-structure data were performed using Kolmogorov-Smirnov one sample tests that assumed standard normal distributions. Differences in chirp-structure parameters between control and androgen-treated fish were determined by independent $t$ tests. Correlations between chirp-structure parameters were determined using Pearson's correlation matrix and Bartlett's $\chi^{2}$ analysis. Cluster analysis and principle component analysis

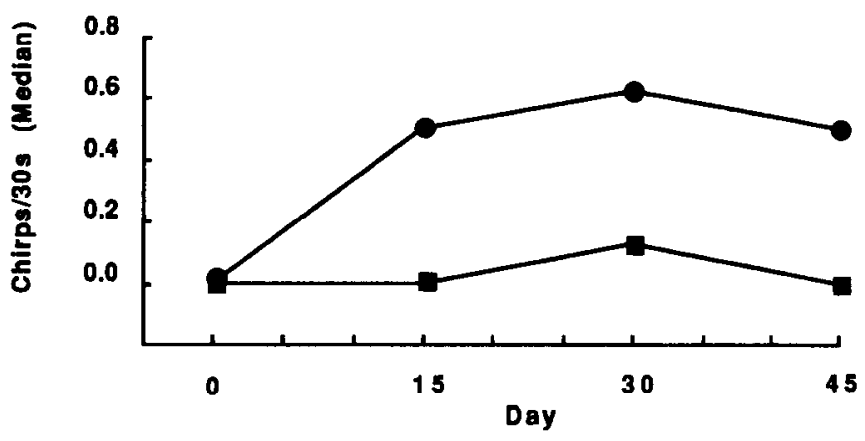

Figure 1. Temporal changes in the number of chirps given by blankimplanted (squares) and androgen-implanted (circles; T and DHT combined) females in response to both the $3 \mathrm{~Hz}$ below and $10 \mathrm{~Hz}$ above stimuli on the days indicated. Each data point represents the median value of 6-11 animals. The maximum ranges of chirp rates recorded from blank-implanted and androgen-implanted females were 0 to 8.5 chirps/30 sec and 0 to $11.0 \mathrm{chirps} / 30 \mathrm{sec}$, respectively.

of chirp-structure data were performed using programs provided in the SYSTAT computer package. Unless otherwise noted, all measures of variation are mean and standard error.

\section{Results}

\section{Hormone induction of chirping in females}

As demonstrated in our previous study (Dulka and Maler, 1994), androgen treatment significantly $(p<0.05)$ increased the proportion of females that chirped in response to the $3 \mathrm{~Hz}$ below and $10 \mathrm{~Hz}$ above stimuli. Chirp responses in all animals were low prior to hormone treatment and did not differ between groups (Fig. 1). Following implantation with blank Silastic capsules, only 3 of 10 control females chirped in response to the stimuli over $45 \mathrm{~d}$ of testing, and median chirp rates in these animals was always below $0.2 \mathrm{chirp} / 30 \mathrm{sec}$ (Fig. 1). In contrast, both $\mathrm{T}$ and DHT were equally effective at increasing chirp responses in females; therefore, the behavioral data collected from T- and DHT-implanted females were combined prior to making statistical comparisons to the controls (Fig. 1). For example, 7 of 10 females implanted with $\mathrm{T}$ and 7 of 12 females implanted with DHT chirped in response to the $3 \mathrm{~Hz}$ below and $10 \mathrm{~Hz}$ above stimuli, and the combined median chirp rate of these animals $(0.5$ chirps $/ 30 \mathrm{sec})$ was significantly $(p<0.05)$ different from the controls on day 15 (Fig. 1). Androgen-treated females continued to give significantly higher chirp rates compared to controls on days 30 and 45 (Fig. 1). Although androgens caused a significant increase in the number of stimulus-evoked chirps, treated females chirped at lower rates than the control group of males (18.6 chirps/30 sec; combined median over all test days; present study).

\section{Androgen-induced changes in chirp structure}

Because females generally do not chirp in responses to the $3 \mathrm{~Hz}$ below or $10 \mathrm{~Hz}$ above stimuli, only a limited number of chirps $(n=47)$ were recorded from nontreated (day 0$)$ and blankimplanted females (controls). Computer analysis of these chirps revealed that they all fell into the same category, in that they were normally distributed for the chirp parameters of AVFREQ and DUR (Fig. 2). Although the analysis indicated that the data for AMP and were not normally distributed, the deviation from normality could be attributed to three outlying data points that skewed the distributions (Fig. 2).

Although the values for each chirp parameter provide a rea- 

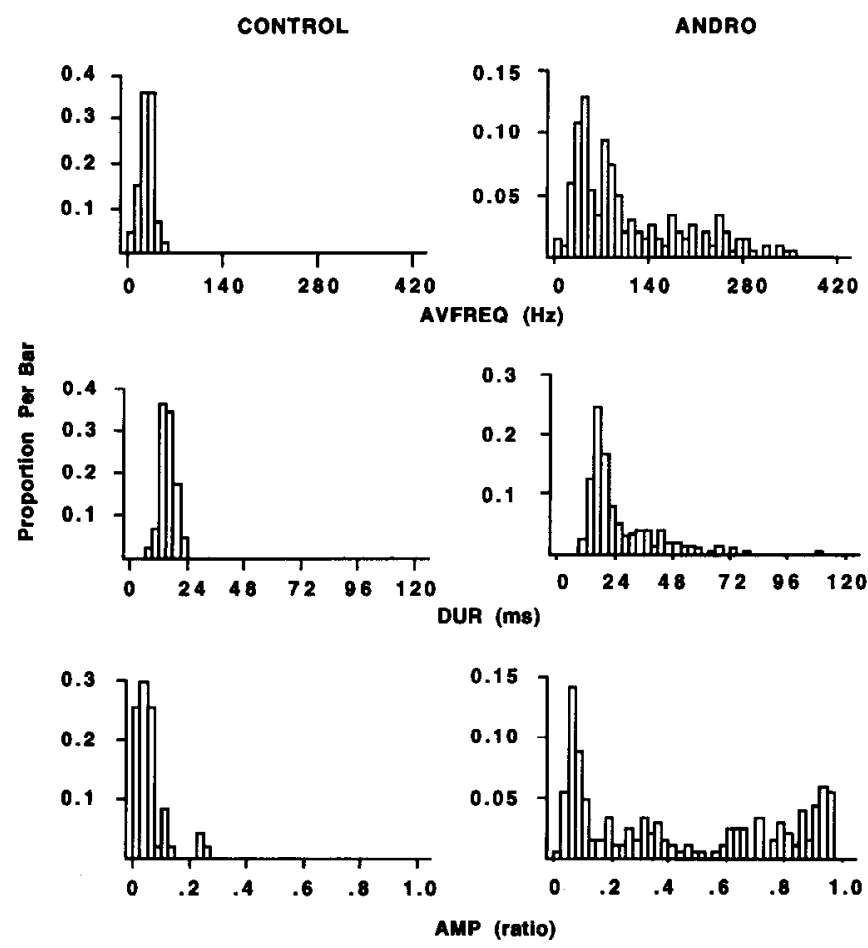

Figure 2. Frequency histograms for the chirp parameters AVFREQ $(\mathrm{Hz})$, DUR (msec) and AMP (ratio) recorded from control and androgen-implanted (ANDRO) females. The control data $(n=47)$ was obtained by combining all chirps recorded from pretreated (day 0 ) and blank-implanted females. The ANDRO data $(n=204)$ was obtained by combining all posttreated chirps recorded from T-implanted and DHTimplanted females.

sonable approximation of chirp structure in control animals, it is important to point out that they over estimate the extent of EOD modulations for each parameter. For example, although all of the EOD modulations recorded on day 0 had the characteristic acoustic quality of chirps, and were sufficient to cause small physiograph pen deflections, not all of these chirps had frequency modulations that were sufficient to trigger data acquisition. Thus, chirps with relatively low frequency modulations (i.e., less than a $20-30 \mathrm{~Hz}$ increase in peak EOD frequency) tended not to be captured and could not be included in the computer analysis. The values reported here for chirp structure in control an- imals are, therefore, conservative estimates because they make it more difficult to detect significant differences in chirp structure between control and androgen-implanted animals.

In contrast to controls, 204 behaviorally evoked chirps were recorded from androgen-implanted females. As in our previous study (Dulka and Maler, 1994), the average height of chirpassociated physiograph pen deflections recorded from androgenimplanted females were larger than those recorded from the controls (data not shown), and this difference appeared to increase over the course of the experiment. Analysis of these chirps revealed non-Gaussian distributions for all three of the chirp parameters, and these were significantly $(p<0.001)$ different from the combined chirps recorded from nontreated (day 0 ) and blank-implanted animals (Fig. 2).

The data on androgen-induced changes in chirp structure are summarized in Figures 2 and 3. We graphically present data on AVFREQ instead of FREQ, since the two are highly correlated $\left(r^{2}=0.8648\right)$ and provide the same type of information: the coefficients of variation for FREQ (0.78) and AVFREQ (0.77) were similar for both treated and nontreated groups, and the ratios of frequency increase during a chirp (3.8 and 3.9 times higher in androgen-implanted females compared to controls for FREQ and AVFREQ, respectively) were also similar for the two parameters. However, AVFREQ is less strongly influenced by transient frequency increases that may occur over 1-2 EOD cycles during a chirp and may therefore more accurately reflect the magnitude of frequency change that normally occurs during a chirp in androgen-implanted animals. The measures of AMP and ABAMP were also highly correlated $\left(r^{2}=0.8493\right)$, and for clarity, we only present data on AMP because both measures of amplitude provide the same type of information. Data on androgen-induced changes in chirp DUR are also provided (Figs. 2 and 3).

Androgen treatment significantly increased the AVFREQ of behaviorally evoked chirps in females. The AVFREQ recorded during chirping from control animals had a mean of $28.1 \pm 1.4$ $\mathrm{Hz}$, and chirps from these animals never had AVFREQs that exceeded $100 \mathrm{~Hz}$ (Fig. 2). In contrast, the AVFREQ of chirps recorded from androgen-implanted females had a mean of 109.3 $\pm 5.9 \mathrm{~Hz}$, and this value was significantly different $(p<0.001)$ from controls; many of the frequency modulations had AVFREQs that exceeded $200-400 \mathrm{~Hz}$ (Fig. 2).

Androgen treatment also increased chirp DUR in females. The
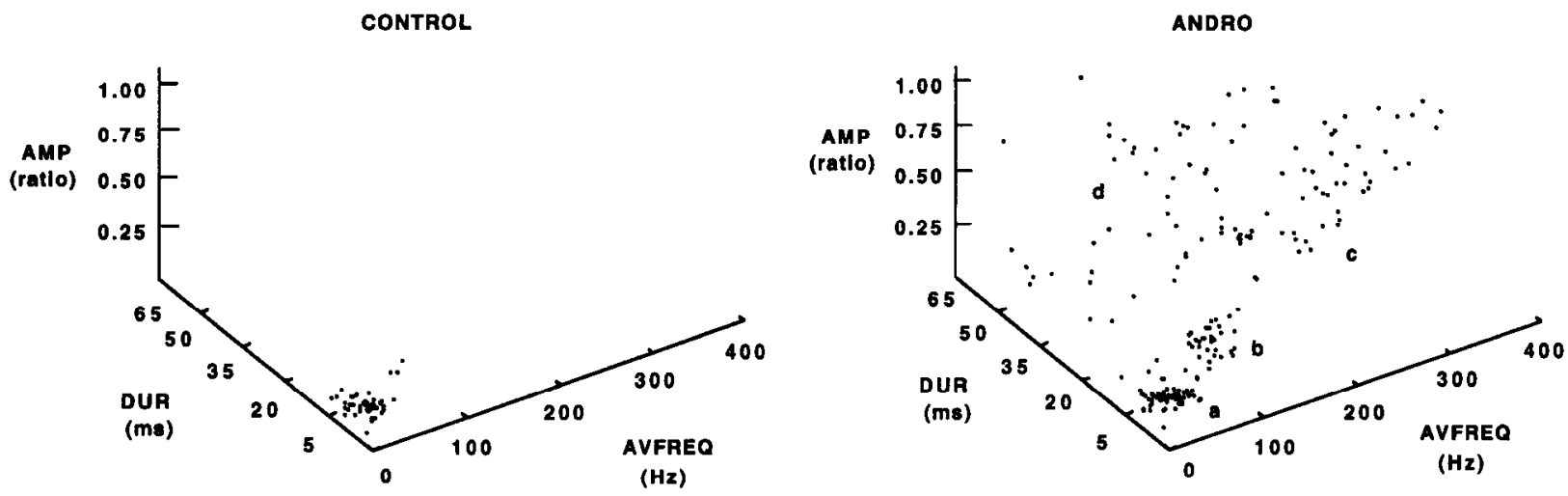

Figure 3. Three-dimensional plots of the chirp parameters AVFREQ ( $H z), D U R$ (msec), and AMP (ratio) recorded from control and androgenimplanted $(A N D R O)$ females. Note that androgen-implanted females produce a mixture of control-like chirps and a variety of chirps with radically different structures. The letters that appear on the ANDRO plot correspond to the type of chirps shown in Figure 4. 
mean DUR of chirps recorded from control animals was 15.7 $\pm 0.4 \mathrm{msec}$, and the upper range for this value never exceeded $24 \mathrm{msec}$ (Fig. 2). The mean DUR of chirps recorded from androgen-implanted females $(26.1 \pm 1.1 \mathrm{msec})$ was significantly different $(p<0.001)$ from controls, and some of these chirps had DURs that exceeded 60-70 msec (Fig. 2).

Chirp AMP was also significantly $(p<0.001)$ affected by androgen treatment. The AMP ratio of chirps recorded from control animals had a mean of $0.057 \pm 0.007$ and all values, except the three outlying data points (see Figs. 2, 3), fell below an AMP value of 0.2 (Fig. 2). In contrast, the AMP ratio of chirps recorded from androgen-implanted animals had a mean of 0.429 \pm 0.024 , and many of these chirp amplitude modulations approached a value of 1.0 , indicating a near total collapse of EOD during a chirp (Fig. 2).

Three dimensional plots of the chirp parameters AVFREQ, DUR, and AMP indicate that $94 \%$ of the chirps recorded from nontreated and blank-implanted females form a tight cluster of data points that are statistically homogenous; the only exception being the three outlying chirps with slightly larger AMPs (Fig. 3 ). These points are within the range of values obtained from males in the present study (male values: AVFREQ $=31.8 \pm$ $5.8 \mathrm{~Hz} ;$ DUR $=18.9 \pm 1.3 \mathrm{msec} ;$ AMP ratio $=0.043 \pm 0.004$ ) and similar to those previously reported for males (i.e., type 1 chirps of Zupanc and Maler, 1994). In contrast, when this pattern of chirp structure is compared to that of the androgen-implanted females, it becomes obvious that the hormone-treated females respond to the behavioral stimuli by giving a mixture of both control-like chirps and a variety of chirps with radically different structures (Fig, 3). Examples of some of the chirps recorded from androgen-implanted females are shown in Figure 4. Although we could not separate these into statistically different categories using cluster analysis, the use of principal component analysis of AVFREQ, DUR, and AMP indicated that two principle components account for $95 \%$ of the variance of the data. The first component is influenced primarily and equally by AVFREQ and AMP (rotated component 1 loadings for AVFREQ, AMP, and DUR were 0.9481, 0.9450, and 0.1236, respectively), while the second component is influenced primar ily by DUR (rotated component 2 loadings for AVFREQ, AMP, and DUR were $0.1077,0.1282$, and 0.9923 , respectively). This is consistent with the high degree of correlation between AVFREQ and AMP $\left(r^{2}=0.7830\right)$ and the low correlation between either of these measures and the DUR of a chirp (AVFREQ vs DUR, $r^{2}=0.2379$; AMP vs DUR, $r^{2}=0.3622$ ). Principle components are orthogonal or unrelated to each other (Harman, 1977). This implies that AVFREQ and AMP are related chirp parameters that theoretically could be regulated by a single androgen-sensitive mechanism. In contrast, because DUR is relatively independent of AVFREQ and AMP, it theoretically could be controlled by a separate androgen-sensitive mechanism.

\section{Androgen-induced changes in SPl-ir in the forebrain}

The Amersham SP antisera produced the same pattern of labeling as previously described and clearly revealed sex differences between males and females (Weld and Maler, 1992). A qualitative ranking of SPl-ir in the forebrain of control and androgen ( $\mathrm{T}$ and DHT)-treated females is provided in Table 1 (nomenclature of Maler et al., 1991). There were no obvious differences in SPl-ir in the telencephalon of control, T-implanted, or DHTimplanted females (Table 1). All three groups showed intense $\mathrm{SPl}-\mathrm{ir}$ in the glomerular layer of the $\mathrm{OB}$, and fibers associated
A AvFreq $=41 \mathrm{~Hz} ;$ Dur $=18 \mathrm{~ms} ; A m p=0.07$

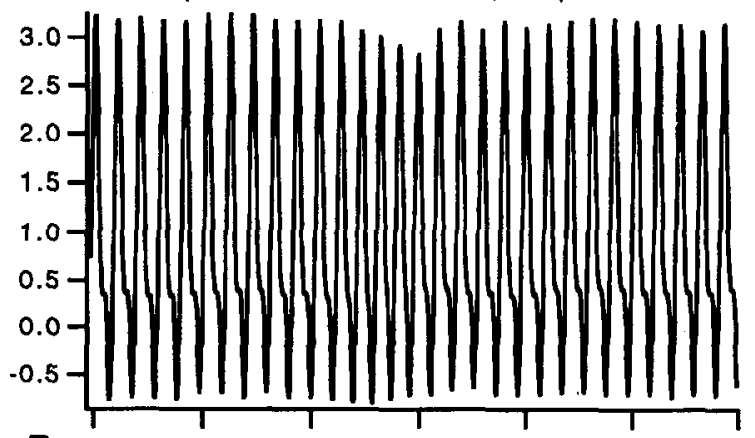

B $\quad$ AvFreq $=87 \mathrm{~Hz}$; Dur $=19 \mathrm{~ms} ; A m p=0.33$
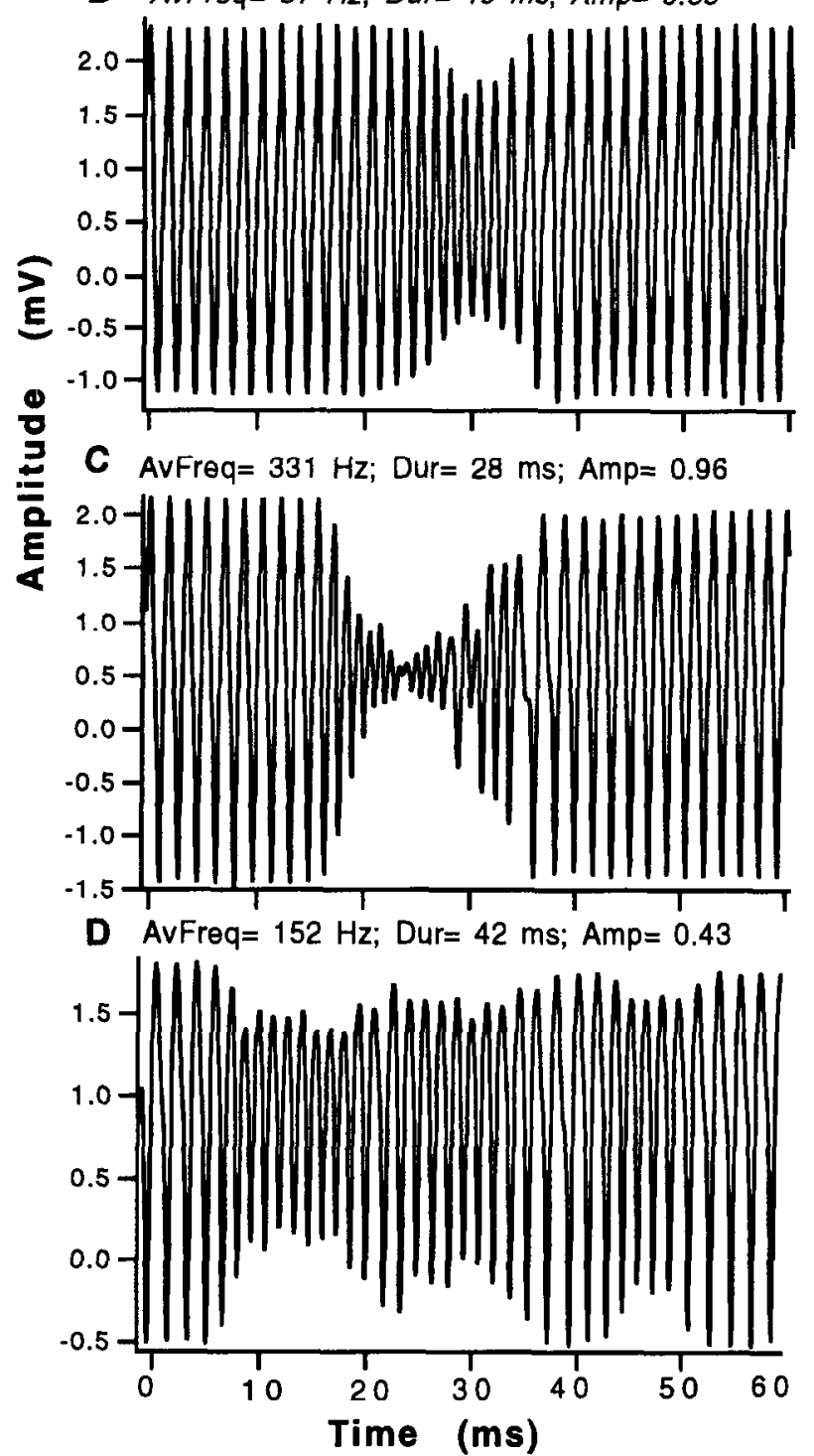

Figure 4. Examples of the various types of chirps rccorded from androgen-implanted females as indicated in Figure 3 . The time base $(60$ $\mathrm{msec}$ ) of the waveforms are all the same so that differences in chirp duration are easily recognizable. Values for the chirp parameters $A V F R E Q, D U R$, and $A M P$ are provided for each example. The chirp seen in $A$ is typical of those produced by control females and nonimplanted males. 


\begin{tabular}{|c|c|c|c|}
\hline & Control & $\mathrm{T}$ & DHT \\
\hline Telencepha & & & \\
\hline $\mathrm{OB}$ & ++ & ++ & ++ \\
\hline Dlv & + & + & + \\
\hline $\mathrm{Vn}$ & + & + & ++ \\
\hline Vv & ++ & ++ & ++ \\
\hline Vl & + & + & + \\
\hline VIr & + & + & + \\
\hline Vs & ++ & ++ & ++ \\
\hline FB & ++ & ++ & ++ \\
\hline MOTF & + & + & + \\
\hline Diencephal & & & \\
\hline $\mathrm{PPa}$ & + & ++ & ++ \\
\hline $\mathrm{CP}$ & - & ++ & ++ \\
\hline PPn-VT & - & ++ & ++ \\
\hline $\mathrm{Ha}$ & - & ++ & ++ \\
\hline $\mathrm{Hv}$ & - & ++ & ++ \\
\hline $\mathrm{Hl}$ & ++ & ++ & ++ \\
\hline $\mathrm{Hc}$ & ++ & ++ & ++ \\
\hline $\mathrm{nRLm} 2$ & + & + & + \\
\hline $\mathrm{nRP}$ & + & + & + \\
\hline SRP & ++ & ++ & ++ \\
\hline
\end{tabular}

Each symbol represents the average pattern of staining observed in four animals; - , complete lack of staining; + , weak to moderate staining; ++ , moderate to strong staining. Abbreviations are listed in the Appendix.

with this system gave rise to weak to moderate staining in the medial olfactory terminal field (MOTF) of the anterior telencephalon (Table 1). SPl-ir staining in all three groups of females was also similar in the ventral (Vv), lateral (V1), intermediate rostral (VIr), nother (Vn), and supracommisural (Vs) subdivisions of the ventral telencephalon (Table 1). These regions were primarily characterized by moderate to strong staining of numerous SPl-ir boutons; few, if any, immunoreactive cell bodies were observed at these levels. A similar pattern of staining was observed in the forebrain bundles and in the ventral subdivision of the dorsal telencephalon (Dlv) in which there were no obvious differences between treatment groups (Table 1, Fig. 5). It is important to point out that SPI-ir staining in the telencephalon is not sexually dimorphic in this species; both males and females show a similar pattern of SPl-ir in all of the brain regions described above (Weld and Maler, 1992), as do the treated and nontreated females in the present study.

In contrast to the telencephalon, numerous structures within the diencephalon of A. leptorhynchus show marked sex differences in SPl-ir. These areas include the anterior subdivision of nucleus preopticus periventricularis ( $\mathrm{PPa}$ ), hypothalamus anterioris $(\mathrm{Ha})$, hypothalamus ventralis $(\mathrm{Hv})$, central-posterior thalamic nucleus (CP), and the prepacemaker nucleus (PPn). All of these regions are densely innervated by SPI-ir in males, but are either weakly stained or completely devoid of SPl-ir in females (Weld and Maler, 1992; present study).

After 30-45 d of implantation with blank capsules, control animals showed the typical female pattern of SPl-ir in the anterior diencephalon (Table 1). SPI-ir was generally weak or absent in the PPa, Ha, Hv, CP, and PPn of blank-implanted females (Figs. 5-8), but clearly present in males (Fig. 8). Control females did, however, show positive SPl-ir staining in regions of the posterior diencephalon that do not appear to be sexually dimorphic (Weld and Maler, 1992). For example, moderate to strongly stained fibers and scattered cell bodies were consistently observed in the hypothalamus lateralis $(\mathrm{HI})$ of control females (Table 1, Fig. 7) and nontreated males (not shown). Both sexes also showed a similar pattern of diffuse SPl-ir boutons in the hypothalamus caudalis $(\mathrm{Hc})$, the second medial subdivision of the nucleus recessus lateralis (NRLm2), and the superior nucleus recessus posterioris (SRP; Table 1). Thus, the lack of SPl-ir staining observed in diencephalic regions ( $\mathrm{PPa}, \mathrm{Ha}, \mathrm{Hv}, \mathrm{CP}$, and PPn) of control females does not appear to be due to technical problems, since SPl-ir was clearly present in the same animals at both more rostral telencephalic and more caudal diencephalic levels.

In contrast to the controls, females implanted with either $\mathrm{T}$ or DHT showed a pronounced increase in the pattern of SPl-ir in the diencephalon within all the sexually dimorphic nuclear
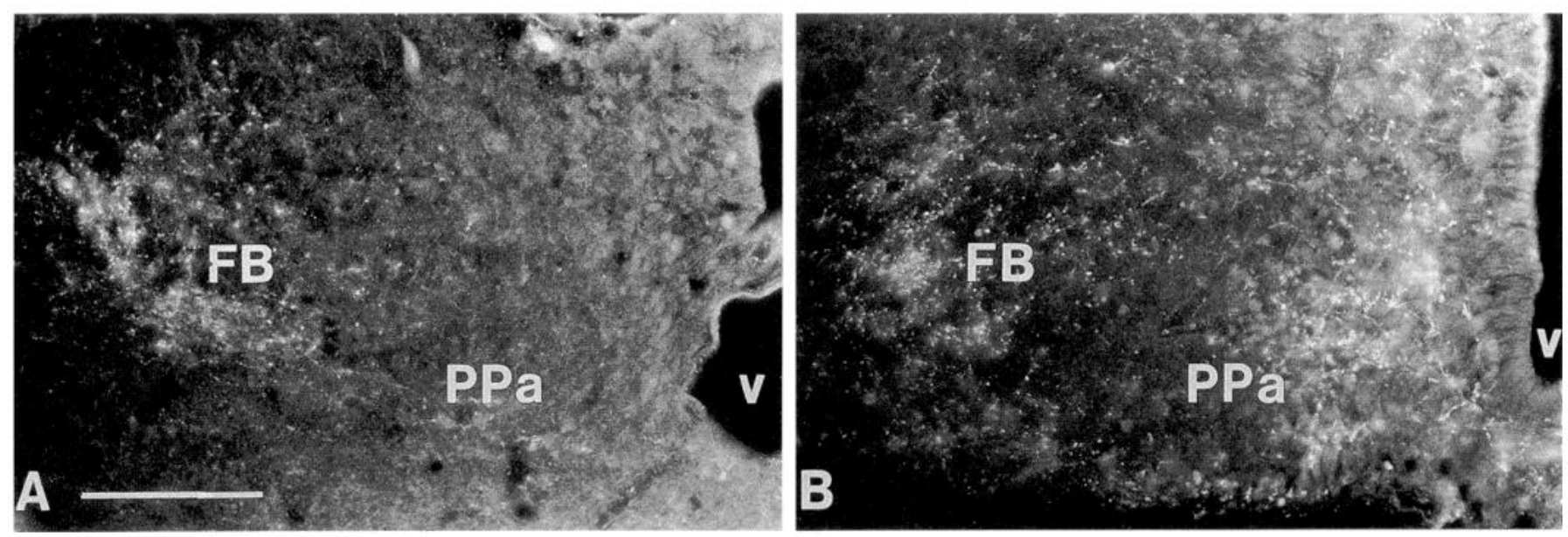

Figure 5. Fluorescence photomicrographs of transverse sections through the nucleus preopticus periventricularis anterioris $(\mathrm{PPa})$ showing the regional distribution of SPI-ir in a blank-implanted control female $(A)$ and a DHT-implanted female $(B, 45 \mathrm{~d}$ posttreatment). The DHT-implanted female $(B)$ shows intense SPl-ir labeling in the PPa; this same area is almost completely devoid of SPl-ir in the control female $(A)$. Note that SPlir is present in the medial forebrain bundles $(F B)$ of both animals. Ventricle $(v)$. Scale bar, $100 \mu \mathrm{m}$. 

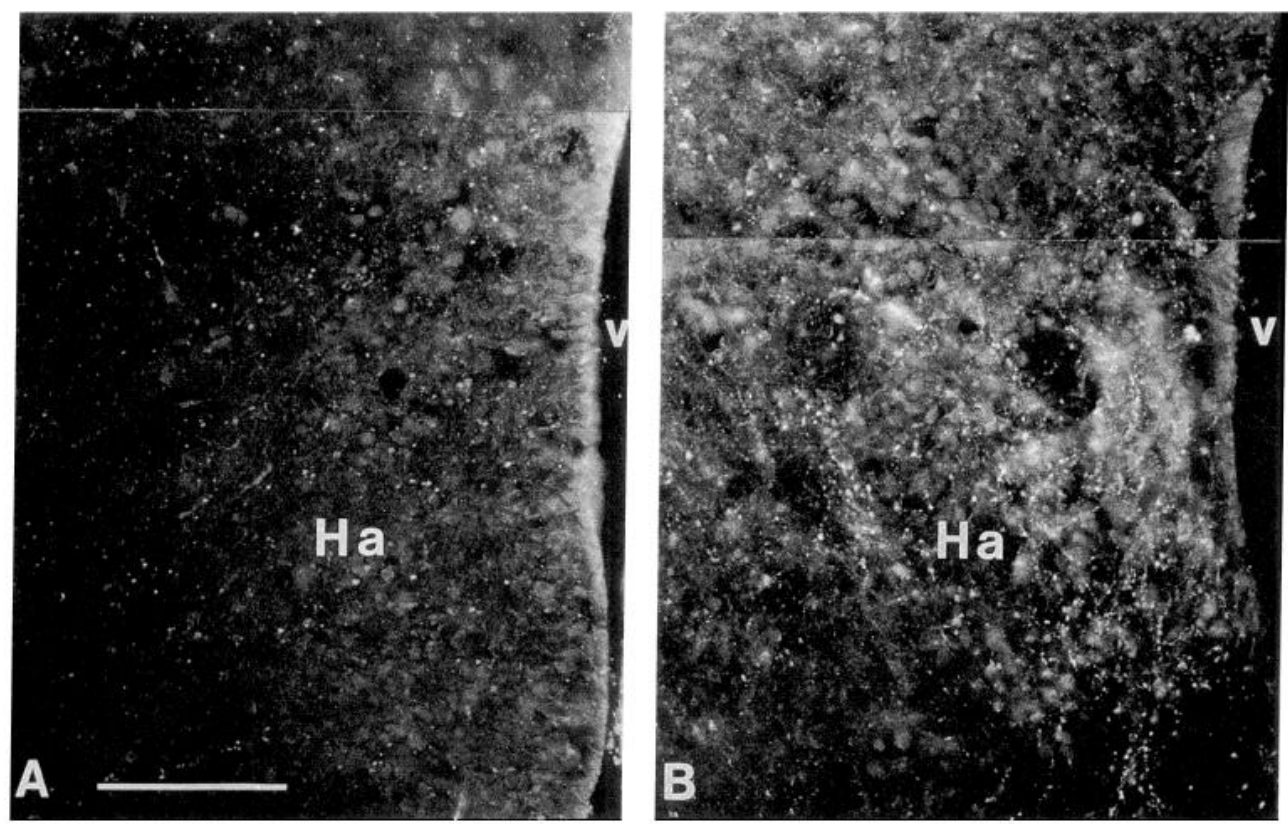

Figure 6. Fluorescence photomicrographs of transverse sections through the rostral portion of the anterior hypothalamic nucleus $(\mathrm{Ha})$ showing the regional distribution of SPl-ir in a blank-implanted control female $(A)$ and a DHT-implanted female ( $B, 45$ d posttreatment). The DHT-implanted female (B) shows intense SPl-ir labeling in the $\mathrm{Ha}$, whereas the same area in the control female $(A)$ is completely devoid of SPI-ir. DHT-implanted females tended to have less SPl-ir in the caudal portion of Ha. Ventricle $(v)$. Scale bar, $100 \mu \mathrm{m}$.

groups (Table 1, Figs. 5-8). Increased numbers of SPl-ir boutons were detected in the PPa, Ha, Hv, CP, and the PPn-VT (Table 1, Figs. 5-8). There were no apparent qualitative differences in SPl-ir staining between T and DHT-implanted females (Table 1). More importantly, SPl-ir did not appear to differ between control and hormone-implanted animals in the telencephalon or posterior diencephalon (Table 1).
Quantitative analysis of the number of SPl-ir particles in the PPn-VT in control and androgen-treated females revealed significant $(p<0.01)$ differences between groups (Fig. 9). On average, the PPn-VT region in T and DHT-implanted females had four times the number of SPl-ir particles observed in the same region of controls (Fig. 9). However, although the hormone treatments caused a pronounced shift in SPl-ir staining in the direc-
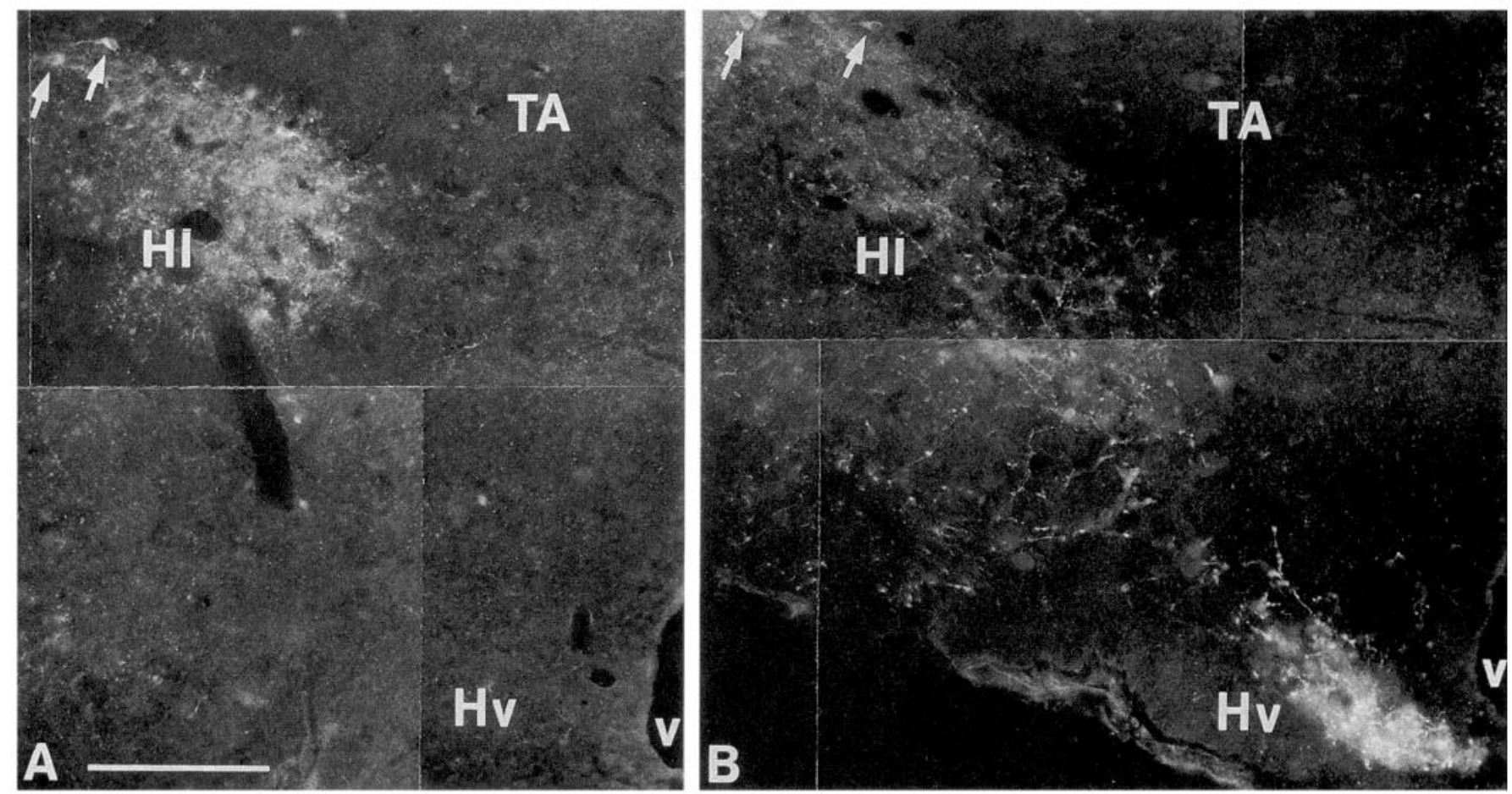

Figure 7. Fluorescence photomicrographs of transverse sections through the ventral hypothalamic nucleus $(H v)$ showing the regional distribution of SPl-ir in a blank-implanted control female $(A)$ and a DHT-implanted female $(B, 45 \mathrm{~d}$ posttreatment). Compared to the control female $(A)$, androgen treatment $(B)$ caused a clear and pronounced induction of SPI-ir in Hv. Note that both animals show weak to moderate labeling of SPIir fibers and cell bodies (arrows) in lateral hypothalamus $(\mathrm{Hl})$. The nucleus tuberis anterior $(\mathrm{TA})$ is devoid of SPl-ir in both cases. Ventricle ( $\mathrm{v}$ ). Scale bar, $100 \mathrm{~mm}$. 

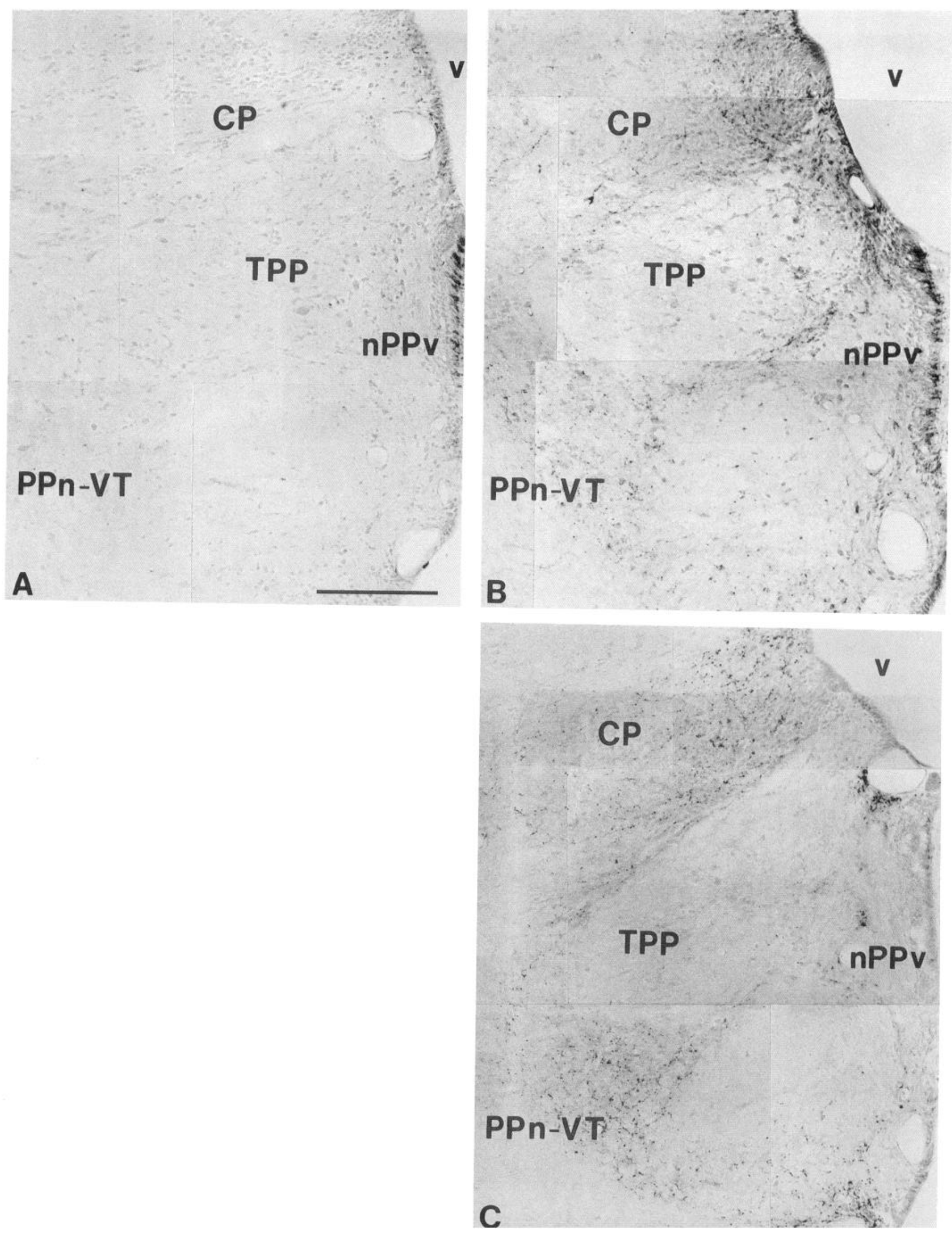
tion of the typical male pattern, the density of SPl-ir did not reached levels normally observed in males (Figs. 8, 9).

\section{Blood levels of immunoreactive testosterone}

At the end of the experiment, all fish were sacrificed and verified to be females. Sexual maturity (gonadosomatic index; ovarian weight/body weight $\times 100$ ) did not differ between groups. T-implanted females had significantly $(p<0.01)$ higher blood levels of $\mathrm{T}(15.86 \pm 3.43 \mathrm{ng} / \mathrm{ml})$ compared to controls $(1.94 \pm 0.72$ $\mathrm{ng} / \mathrm{ml})$. The $\mathrm{T}$ antiserum recognized significantly $(p<0.01)$ more immunoreactive androgens in the blood of DHT-implanted females $(6.49 \pm 1.07 \mathrm{ng} / \mathrm{ml})$ compared to the blank-implanted controls. The nontreated males that were included in the study had low levels of circulating $\mathrm{T}(0.76 \pm 0.14 \mathrm{ng} / \mathrm{ml})$ compared to the blank-implanted and androgen-treated females.

\section{Discussion}

The present results are consistent with our previous report on the hormonal induction of chirping behavior in female A. leptorhynchus (Dulka and Maler, 1994). As before, increased blood levels of androgens caused increased chirping in females. In addition, the present results confirm our previous predictions (Dulka and Maler, 1994) that androgen treatment leads to a gradual change in chirp structure in females. This study also presents new information on one mechanism by which androgens might act centrally to modulate female chirping activity; namely, androgen-induced changes in SPl-ir that are directly correlated with hormone-induced changes in chirping behavior. Moreover, the androgen-induced increases in SPl-ir occurred in regions known to be sexually dimorphic in this species (Weld and Maler, 1992). The latter relationship may be important for understand ing the neural and hormonal regulation of chirping, since males readily chirp in response to appropriate electrosensory stimuli, whereas females normally do not (Dye, 1987; Dulka and Maler, 1994; Zupanc and Maler, 1994).

\section{Androgen-induced changes in SPl-ir}

The androgen-induced changes in SPl-ir appear specific since only diencephalic brain regions known to be sexually dimorphic for the peptide were affected by the hormone treatments. Although we did not attempt to quantify SPl-ir in the telencephalon (Vv, Vs, FB, and MOTF) or caudal diencephalon (Hl, nRLM2, and SRP), we could not see any qualitative differences between treated and nontreated females. In contrast, the androgen-induced changes in SPl-ir observed in the diencephalon (PPa, Ha, $\mathrm{Hv}, \mathrm{CP}$, and PPn-VT) were striking and clearly different from the normal female pattern. Interestingly, all of these diencephalic regions have moderate (PPn-VT and $\mathrm{CP}$ ) to high ( $\mathrm{PPa}, \mathrm{Ha}$, and $\mathrm{Hv}$ ) densities of tachykinin binding sites (NK1); the regional distribution of these binding sites does not appear to be sexually dimorphic (Weld et al., 1994). Thus, androgen-induced increases in SPl-ir may exert physiological actions at these diencephalic sites.

Our quantitative analysis focused only on the PPn, since this

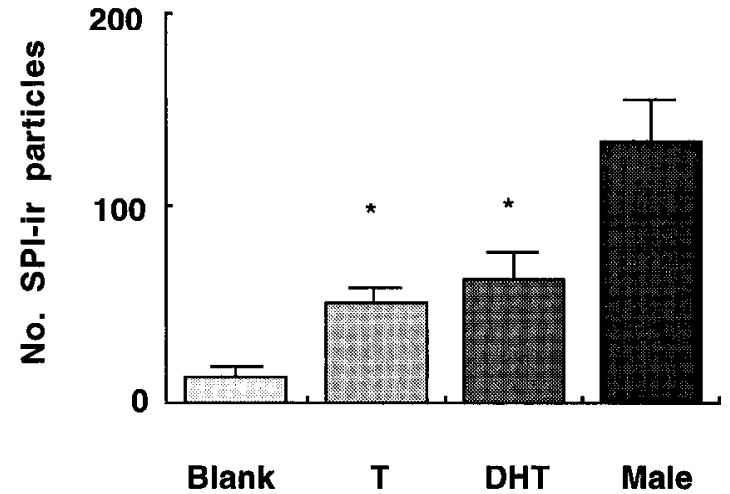

Figure 9. Quantification of SPl-ir particles in the PPn-VT of blankimplanted $(n=8)$, T-implanted $(n=8)$, and DHT-implanted females $(n=11)$, and in normal males $(n=6)$. Each bar represents the mean and standard error for each group. Asterisks indicate significant $(p<$ 0.05 ) differences compared to the blank-implanted control group.

region is known to function as a command center for chirping behavior in this animal (Kawasaki et al., 1988). A second prepacemaker region, the sublemniscal prepacemaker nucleus, has recently been described (Keller et al., 1991; Metzner, 1992). However, this region is devoid of SPl-ir in males and females (Weld and Maler, 1992) and in androgen-treated females (present study); it also appears to lack tachykinin binding sites (Weld et al., 1994). Thus, androgen-induced changes in SPl-ir may effect electrocommunication only via the chirp subdivision of the PPn (see Kawasaki et al., 1988, for anatomical and functional subdivisions of the PPn).

All of the diencephalic regions that showed differences in SP1ir (PPa, Ha, Hv, CP, and PPn) have been implicated in the control of reproductive or endocrine function in teleosts. For example, the PPa regulates sexual behavior (Kyle et al., 1982), gamete release (Demski and Hornby, 1982) and pituitary function (Peter et al., 1990). The PPn controls chirping activity (Kawasaki et al., 1988) and CP is known to regulate sex role-associated color change and gamete release (Demski and Dulka, 1986). In addition, $\mathrm{PPa}, \mathrm{Ha}, \mathrm{Hv}$, and $\mathrm{CP}$ innervate the pituitary (Johnston and Maler, 1992). The present results suggest that androgens cause both specific and widespread changes in SPl-ir that relate to a functional system that interrelates sexual behavior and pituitary function.

The steroid-induced changes in SPl-ir described here are similar to those observed in rodents and birds. It is well documented that SP and/or related tachykinins are sexually dimorphic (Frankfurt et al., 1985; Malsbury and McKay, 1987, 1989), show estrous cycle variations (Frankfurt et al., 1986), and undergo changes in staining following hormonal treatment (Blaustein et al., 1991; Swann and Newman, 1992; Akesson, 1994). Brain areas that show SPl-ir include the anterior pituitary, ventromedial nucleus of the hypothalamus, medial nucleus of the amygdala, bed nucleus of the stria terminalis, and the medial preoptic area, all of which mediate steroidal induction of reproductive

$\leftarrow$

Figure 8. Transverse photomicrographs of SPl-ir (peroxidase reacted material) in the region of the ventral dendritic territory of the prepacemaker nucleus $(P P n-V T)$ in a blank-implanted female $(A)$, a DHT-implanted female $(B, 30 \mathrm{~d}$ posttreatment), and a normal male $(C)$. The central posterior nucleus $(C P)$ and PPn-VT regions of the control female $(A)$ are completely devoid of SPl-ir, even though the nucleus posterioris periventricularis $(n P P v)$ shows positive staining. In contrast, the DHT-implanted female $(B)$ shows a clear induction of SPl-ir in the PPn-VT, and to a lesser extent, in CP as well. Note that the pattern of SPl-ir staining in the DHT-implanted female $(B)$ approaches that normally observed in males $(C)$, 'The periventricular nucleus of the tuberculum $(T P P)$ shows negative staining in each case. Ventricle $(v)$. Scale bar, $100 \mu \mathrm{m}$. 


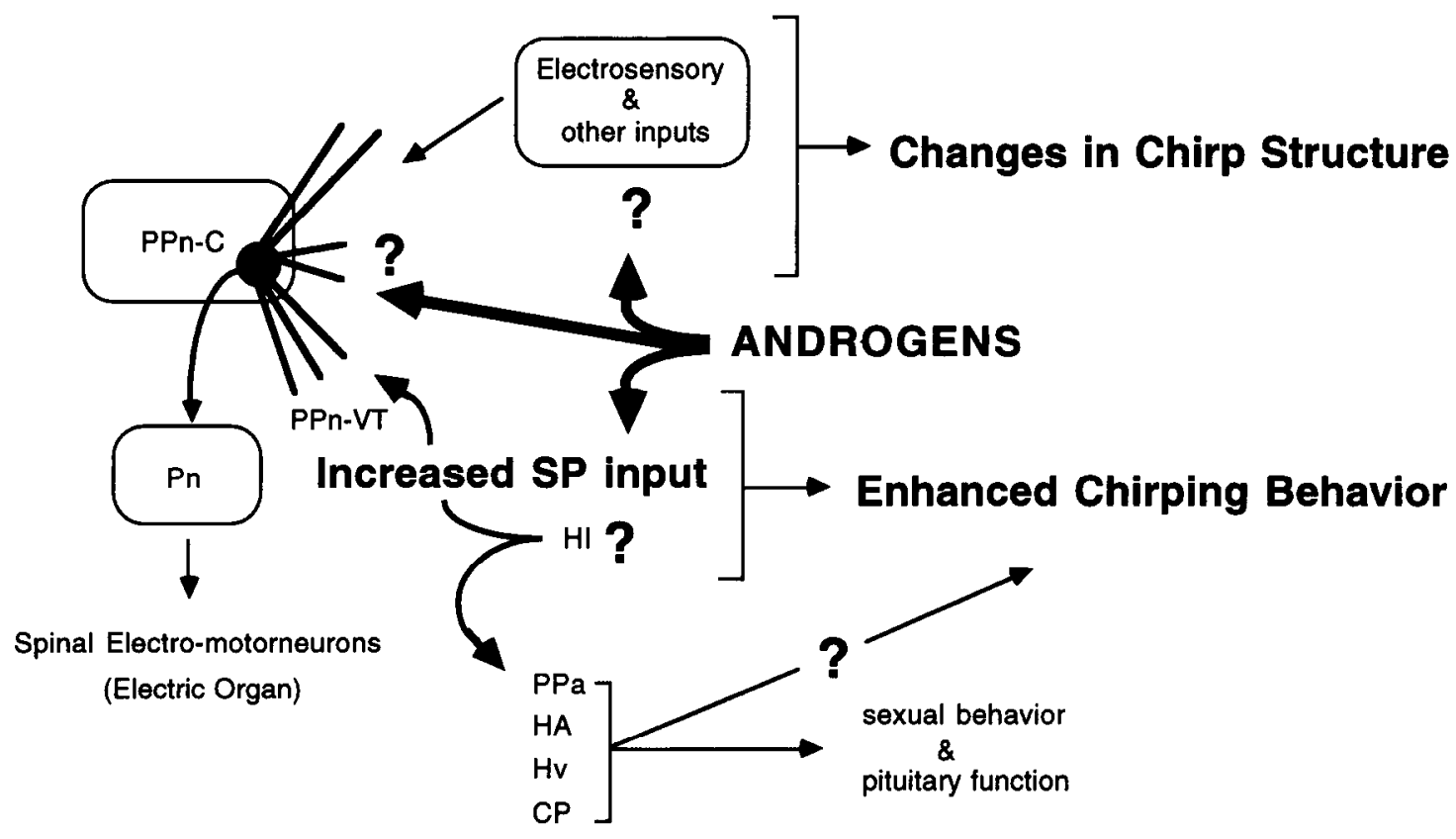

Figure 10. Summary diagram of the possible mechanisms by which androgens could influence female chirping behavior in A. leptorhynchus. Increased SPl-ir input to the PPn-VT is correlated with androgen-induced chirping behavior in females. Androgens also increase SPl-ir input to diencephalic regions $(\mathrm{PPa}, \mathrm{Ha}, \mathrm{Hv}$, and $\mathrm{CP})$ known to regulate sexual behavior and pituitary function; it is not known if these regions also influence chirping behavior. Androgens may effect SPl-ir by acting at the level of $\mathrm{Hl}$, the most likely source of this sexually dimorphic peptide (Weld and Maler, 1992). However, although increased SPl-ir input to the chirp subdivision of the PPn $(P P n-C)$ may account for the induction of female chirping behavior, it does not appear to be responsible for causing concomitant changes in chirp structure. Androgen-induced changes in chirp structure may be mediated by other neuropeptide inputs to the PPn-C, or through direct hormonal affects on electrosensory nuclei that project to the PPn-C (Heiligenberg et al., 1991; Metzner et al., 1991). Alternatively, androgens may modulate chirping activity by directly altering the excitability, morphology, and/or connectivity of PPn-C neurons (Zupanc and Heiligenberg, 1989). In addition, it is also possible that androgens modify chirping by affecting the membrane properties of the pacemaker nucleus ( $P n$; Meyer, 1984), and the spinal motor neurons that form the electric organ in this species.

behavior. In addition, androgen receptors and SPl-ir are colocalized in most of these regions (Newton et al., 1993). In some cases, gonadal steroids regulate SP-tachykinnin-ir in females, but not males (Akesson, 1992). Moreover, the hormonal regulation of sex differences in preprotachykinnin gene expression has been demonstrated (Romano et al., 1989, 1990; Brown et al., 1990; Jonassen and Leeman, 1991).

Exogenously administered SP is effective at triggering various aspects of reproductive behavior. Intracerebral injections of SP evoke male copulatory behavior and facilitate estrogen-induced lordosis behavior in rats (Dornan and Malsbury, 1989; Dornan et al., 1990). Similarly, injections of SP into the PPn triggers chirping behavior in A. leptorhynchus (Weld and Maler, 1991). Taken together, teleosts and other vertebrates appear to share strong relationships between SP and steroid-sensitive systems that govern sexual activity.

The effects we observe appear to be mediated by direct "androgenic" effects, since both $\mathrm{T}$ (an aromatizable androgen) and DHT (a nonaromatizable androgen) were equally effective in modulating chirping behavior and SPl-ir staining. However, at present, we cannot comment on hormonal specificity or androgenic effects on other neuropeptide systems. We also have not identified the source of the androgen-sensitive SPl-ir fibers to the diencephalon, although it is likely that they originate from cells in $\mathrm{Hl}$ (Weld and Maler, 1992). The most parsimonious explanation of our data is that a single population of androgensensilive SPI-ir neurons provides innervation to all the sexually dimorphic diencephalic nuclei. Similar hypotheses has been made for other peptide systems in teleosts (Yu et al., 1987; Oka and Matsushima, 1993).

\section{Androgen-induced changes in chirp structure}

Increased blood levels of androgens clearly effect the type of chirps produced by hormone-treated females. Although androgen-implanted females still give control-like chirps, they also respond by producing very different chirps. In general, androgens increase the frequency, duration, and amplitude ratio of evoked chirps. Principle component analysis suggests that androgens may independently regulate the duration of a chirp versus its frequency and amplitude; however, it is not clear whether this is dependent on increased SP input to the PPn, or whether other mechanisms are involved (see Fig. 10).

Recent studies in a related gymnotiform species, Eigenmannia virescens, have demonstrated that the electrosensory system is capable of detecting chirps (Heiligenberg et al., 1991; Metzner and Heiligenberg, 1991). These studies suggest that the electrosensory system can distinguish between chirps with small versus large amplitude modulations or short versus long durations. It is, therefore, possible that the androgen-induced change in female chirp quality may serve to signal the hormonal status of a female to conspecifics; the utility of such information for the regulation and synchronization of reproductive behavior is obvious. Interestingly, both $A$. leptorhynchus and $E$. virescens produce short and long chirps in a context-specific manner. Chirps of short duration are produced primarily during aggressive interactions, while longer chirps occur more frequently during 
courtship and spawning (Hopkins, 1974; Hagedorn and Heiligenberg, 1985). Although the large amplitude chirps that occur during courtship and spawning (Hagedorn and Heiligenberg, 1985) resemble some of the androgen-induced chirps, it remains to be determined whether androgen treatment is mimicking changes in chirp structure that normally occur during the reproductive season. However, because androgen treatment causes concomitant changes in other electric behaviors, in a direction expected from spawning individuals (Dulka and Maler, 1994), it is likely that some of the differences in chirp structure relate to reproduction. Detailed behavioral and electrophysiological studies will be required to determine the biological significance of various types of chirps, and whether chirp amplitude and duration can provide different information to a recipient fish.

At present, we do not understand why females chirp less that males. It is also not clear if female chirp rates ultimately reach male levels, since this has not been established under natural conditions. For example, although Hagedorn and Heiligenberg (1985) and Hagedorn (1986) described female chirping in a reproductive context, they did not comment on female chirp rates relative to males. In addition, sex differences in chirp rate cannot be explaincd by corresponding differences in circulating levels of $\mathrm{T}$, since males have considerably lower levels of $\mathrm{T}(0.76 \pm$ $0.14 \mathrm{ng} / \mathrm{ml})$ than blank-implanted females $(1.94 \pm 0.72 \mathrm{ng} / \mathrm{ml})$. However, it is possible that male-specific androgens play a role in establishing male chirp rates. For example, 11-ketotestosterone is the predominant blood androgen in most male teleosts (Idler et al., 1960), and is present in higher concentrations than $\mathrm{T}$ in at least one species of electric fish, Sternopygus macrurus (Zakon et al., 1990, 1991). Thus, it remains to be determined if male chirping is regulated by a hormonal mechanism involving 11-ketotestosterone, or whether other factors are involved.

In contrast, $\mathrm{T}$ is known to function as an important female hormone in many freshwater teleosts, especially during the reproductive period (Kagawa et al., 1983; Kobayashi et al., 1986). Indeed, blood levels of $\mathrm{T}$ in female Sternopygus are often higher than those of estradiol and can exceed $\mathrm{T}$ concentrations in males during the rcproductive season (Zakon et al., 1991). The results from this and our previous study (Dulka and Maler, 1994) indicate that a similar situation may exist in A. leptorhynchus. A hormonally mediated mechanism for chirping behavior may allow females to chirp in a reproductive context only when sufficient amounts of $T$ are in the blood. Thus, $T$ may serve as an important hormonal requirement for the synchronization of chirping behavior with discrete reproductive events such as ovulation and spawning (discussed in Dulka and Maler, 1994).

We assume that androgen-induced changes in SPl-ir to the PPn may underly behavioral changes in female chirping activity. However, although this species exhibits clear sex differences in both chirping behavior and SPl-ir staining, the androgen-induced increases in SPl-ir alone cannot account for all the behavioral changes seen in treated females. For example, although SPl-ir is more pronounced in males than in females, males rarely, if ever, give chirps with high frequencies, long durations, and large amplitude modulations (Zupanc and Maler, 1994; Dulka and Maler, 1994; this study). However, control females with little or no SPIir in the PPn-VT rarely, if ever, chirp when tested under the same conditions (Dulka and Maler, 1994; present study). Therefore, androgen-induced increases in SPl-ir input to the PPn-VT may be mainly responsible for the induction of female chirping behavior. In contrast, concomitant changes in chirp structure may be mediated by a separate physiological mechanism, pos- sibly involving the actions of different neurotransmitters or direct hormonal effects on the neural circuitry that controls chirping. A summary of possible mechanisms by which androgens could influence both chirp propensity and structure in females is provided in Figure 10. Although these possibilities remain speculative, they serve as likely starting points for future experiments on the hormonal modulation of chirping behavior in $A$. leptorhynchus.

\section{Appendix}

List of anatomical abbreviations

$\mathrm{CP} \quad$ central-posterior thalamic nucleus

Dlv dorsal telencephalon, ventral subdivision

FB forebrain bundle

$\mathrm{Ha} \quad$ hypothalamus anterioris

$\mathrm{Hc} \quad$ hypothalamus caudalis

$\mathrm{Hl}$ hypothalamus lateralis

Hv hypothalamus ventralis

MOTF medial olfactory terminal field

$\mathrm{nPPv} \quad$ nucleus posterioris periventricularis

nRLm2 nucleus recessus lateralis, medial subdivision 2

$\mathrm{nRLm} 3$ nucleus recessus lateralis, medial subdivision 3

nRP nucleus recessus posterioris

OB olfactory bulb

$\mathrm{PPa} \quad$ nucleus preopticus periventricularis, anterior subdivision

PPn prepacemaker nucleus

PPn-VT prepacemaker nucleus, ventral dendritic territory

SRP superior nucleus recessus posterioris

TA nucleus tuberis anterior

TPP periventrivular nucleus of the tuberculum

$\mathrm{v} \quad$ ventricle: all levels

VIr ventral telencephalon, intermediate rostral subdivision

Vl ventral telencephalon, lateral subdivision

Vn ventral telencephalon, nother subdivision

Vs ventral telencephalon, supracommissural subdivision

$\mathrm{Vv} \quad$ ventral telencephalon, ventral subdivision

\section{References}

Akesson TR (1992) Gonadal steroids regulate immunoreactive tachykinnin in the ventromedial nucleus of the female but not the male rat. Soc Neurosci Abstr 18:370.1.

Akesson TR (1994) Gonadal steroids regulate immunoreactive tachykinnin in the ventromedial nucleus of the rat hypothalamus. J Comp Neurol 341:351-356.

Blaustein JD, Nielsen KH, Delville Y, Turcotte JC, Olster DH (1991) Neuroanatomical relationships of substance $P$ and sex steroid-sensitive neurons involved in sexual behavior. Ann NY Acad Sci 632: 314-331

Breedlove SM (1992) Sexual dimorphism in the vertebrate nervous system. J Neurosci 12:4133-4142.

Brown ER, Harlan RE, Krause JE (1990) Gonadal steroid regulation of substance $\mathrm{P}$ (SP) and SP-encoding messenger ribonucleic acids in the rat anterior pituitary and hypothalamus. Endocrinology 126:330340.

Bullock TH, Heiligenberg W (eds) (1986) Electroreception. New York: Wiley.

Demski LS, Dulka JG (1986) Thalamic stimulation evokes sex-color change and gamete release in a vertebrate hermaphrodite. Experientia 42:1285-1287.

Demski LS, Hornby PJ (1982) Hormonal control of fish reproductive behavior: brain-gonadal steroid interactions. Can J Fish Aquat Sci $39: 36-47$

Dornan WA, Malsbury CW (1989) Peptidgeric control of male rat sexual behavior: the effects of intracerebral injections of substance $P$ and cholecystokinnin. Physiol Bchav 46:547-556.

Dornan WA, Akesson TR, Micevych PE (1990) A substance P projection from the VMH to the dorsal midbrain central gray: implication for lordosis. Brain Res Bull 25:791-796.

Dulka JG, Maler L (1994) Testosterone modulates female chirping behavior in the weakly electric fish, Apteronotus leptorhynchus. J Comp Physiol [A] 174:331-343.

Dye J (1987) Dynamics and stimulus-dependence of pacemaker control 
during behavioral modulations in the weakly electric fish, Apteronotus. J Comp Physiol [A] 161:175-185.

Frankfurt M, Siegel RA, Sim I, Wuttke W (1985) Cholecystokinnin and substance $\mathbf{P}$ concentrations in discrete areas of the rat brain: sex differences. Brain Res 358:53-58.

Frankfurt M, Siegel RA, Sim I, Wuttke W (1986) Estrous cycle variations in cholecystokinnin and substance $P$ concentrations in discrete areas of the rat brain. Neuroendocrinology 42:226-231.

Hagedorn M (1986) The ecology, courtship and mating of gymnotiform electric fish. In: Electroreception (Bullock TH, Heiligenberg W, eds), pp 497-525. New York: Wiley.

Hagedorn M, Heiligenberg W (1985) Court and spark: electric signals in the courtship and mating of gymnotoid tish. Anim Behav 33:254265.

Harman HH (1977) Modern factor analysis, 3d ed. Chicago: University of Chicago Press.

Heiligenberg W (1991) Neural nets in electric fish. Cambridge, MA: MIT Press.

Heiligenberg W, Keller CF, Metzner W, Kawasaki M (1991) Structure and function of neurons in the complex of the nucleus electrosensorius of the gymnotiform fish Eigenmannia: delection and processing of electric signals in social communication. J Comp Physiol [A] 169:151-164

Hopkins CD (1974) Electric communication: functions in the social behavior of Eigenmannia virescens. Behavior 50:270-305.

Hutchison JB (1991) Hormonal control of behavior: steroid action in the brain. Curr Opin Neurobiol 1:562-570.

Idler DR, Scmidt PD, Ronald AP (1960) Isolation and identification of 11 ketotestosterone in salmon plasma. Can J Biochem Physiol 38: $1053-1058$.

Johnston SA, Maler L (1992) Anatomical organization of the hypophysiotrophic systems in the electric fish, Apteronotus leptorhynchus J Comp Neurol 317:421-437.

Jonassen JA, Leeman SE (1991) Developmental and hormonal regulation of the sex difference in preprotachykinnin gene expression in rat anterior pituitaries. Ann NY Acad Sci 632:1-9.

Kagawa H, Young G, Nagahama Y (1983) Changes in plasma steroid hormone levels during gonadal maturation in goldfish Carassius auratus. Bull Jpn Soc Sci Fish 49:1783-1787.

Kawasaki M, Maler L, Rose GJ, Heiligenberg W (1988) Anatomical and functional organization of the prepacemaker nucleus in gymnotiform electric fish: the accommodation of two behaviors in one nucleus. J Comp Neurol 276:113-131.

Keller CH, Kawasaki M, Heiligenberg W (1991) The control of pacemaker modulations for social communication in the weakly electric fish Sternopygus. J Comp Physiol [A] 169:441-450.

Kobayashi M, Aida K, Hanyu I (1986) Annual changes in plasma levels of gonadotropin and steroid hormones in goldfish. Bull Jpn Soc Sci Fish 52:1153-1158.

Kyle AL, Stacey NE, Peter RE (1982) Ventral telencephalic lesions: effects on bisexual behavior, activity and olfaction in the male goldfish. Behav Neural Biol 36:229-241.

Lannoo MJ, Ross L, Maler L, Hawkes R (1991) Development of the cerebellum and its extracerebellar purkinje cell projection in teleost fishes as determined by zebrin II immunocytochemistry. Prog Neurobiol 37:329-363.

MacLusky NJ, Naftolin F (1981) Sexual differentiation of the central nervous system. Science 211:1294-1303.

Maler L, Sas E, Johnston S, Ellis W (1991) An atlas of the brain of the electric fish Apteronotus leptorhynchus. J Chem Neuroanat 4:138.

Malsbury CW, McKay K (1987) A sex difference in the pattern of substance P-like immunoreactivity in the bed nucleus of the stria terminalis. Brain Res 420:365-370.

Malsbury CW, McKay K (1989) Sex differences in the substance P-immunoreactive innervation of the medial nucleus of the amygdala Brain Res Bull 23:561-567.

McEwan BS (1981) Neural gonadal steroid actions. Science 211:13031311.

Metzner W (1992) The sublemniscal prepacemaker nucleus (SPPn) modulates the frequency of the medullary pacemaker nucleus in the gymnotiform fish, Eigenmannia. Soc Neurosci Abstr 18:149:9.

Metzner W, Heiligenberg W (1991) The coding of signals in the electric communication of the gymnotiform fish, Eigenmannia: from electroreceptors to neurons in the torus semicircularis of the midbrain. J Comp Physiol [A] 169:135-150.

Meyer JH (1984) Steroid influences upon discharge frequencies of intact and isolated pacemakers of weakly electric fish. J Comp Physiol [A] 154:659-668

Newton CB, Khan MB, Powell K, Swann JM (1993) Androgen receptor and substance $\mathrm{P}$ are colocalized in the neurons of BNSTpm, Mp and MPNmgn in the male Syrian hamster. Soc Neurosci Abstr 19: 301.4 .

Oka Y, Matsushima T (1993) Gonadotropin-releasing homnone (GnRH)-immunoreactive terminal nerve cells have intrinsic rhythmicity and project widely in the brain. $J$ Neurosci $13: 2161-2176$.

Peter RE, Yu KL, Marchant TA, Rosenblum PM (1990) Direct neural regulation of the teleost adenohypophysis. J Exp Zool Suppl 4:8489.

Romano GJ, Bonner TI, Pfaff DW (1989) Preprotachykinnin gene expression in the mediobasal hypothalamus of estrogen-treated and ovariectomized control rats. Exp Brain Res 76:21-26.

Romano GJ, Mobbs CV, Lauber A, Howells RD, Pfaff DW (1990) Differential regulation of proenkephalin gene expression by estrogen in the ventromedial hypothalamus of male and female rats: implications for the molecular basis of a sexually differentiated behavior. Brain Res 536:63-68

Swann JM, Newman SW (1992) Testosterone regulates substance P within neurons of the medial nucleus of the amygdala, the bed nucleus of the stria terminalis and the medial preoptic area of the male golden hamster. Brain Res 590:18-28.

Van Der Kraak G, Dye HM, Donaldson EM (1984) Effects of LH-RH and des Gly ${ }^{10}\left[\mathrm{D}-\mathrm{Ala}^{6}\right] \mathrm{LH}-\mathrm{RH}-\mathrm{ethylamide}$ on plasma sex steroid profiles in adult female coho salmon (Oncorhynchus kisutch). Gen Comp Endocrinol 55:36-45.

Weld MM, Maler L (1991) Sexually dimorphic distribution of substance $\mathrm{P}$ and its role in the regulation of communication in an electric fish. Soc Neurosci Abstr 17:560.12.

Weld MM, Maler L (1992) Substance P-like immunoreactivity in the brain of the gymnotiform fish Apteronotus leptorhynchus: presence of sex differences. J Chem Neuroanat 5:107-129.

Weld MM, Kar S, Maler L, Quirion R (1994) The distribution of tachykinin binding sites in the brain of an electric fish (Apteronotus leptorhynchus). J Chem Neuroanat, in press.

Yu KL, Nahorniak CS, Peter RE, Corrigan A, Rivier JE, Vale WW (1987) Brain distribution of radioimmunoassayable gonadotropin-releasing hormone in female goldfish: seasonal variation and preovulatory changes. Gen Comp Endocrinol 67:234-246.

Zakon HH, Yan H-Y, Thomas P (1990) Human chorionic gonadotropin-induced shifts in the electrosensory system of the weakly electric fish, Sternopygus. J Neurobiol 21:826-833.

Zakon HH, Thomas P, Yan H-Y (1991) Electric organ discharge frequency and plasma sex steroid levels during gonadal recrudescence in a natural population of the weakly electric fish Sternopygus ma crurus. J Comp Physiol [A] 169:493-499.

Zupanc GKH, Maler L (1994) Evoked chirping in the weakly electric fish, Apteronotus leptorhynchus. J Can Zool 71:2301-2310. 